\section{Emotion recognition and genetic vulnerability}

\section{to schizophrenia}

\author{
BENOIT BEDIOU, FATIMA ASRI, JEROME BRUNELIN, \\ PIERRE KROLAK-SALMON, THIERRY D'AMATO, MOHAMED SAOUD \\ and IMANE TAZI
}

\begin{abstract}
Background Epidemiological studies of schizophrenia suggest that this disorder has a substantial genetic component.

Cognitive and social abilities, as well as the volumes of brain regions involved in emotion processing, have been found to be distributed along a continuum when comparing patients, siblings and controls, with siblings showing intermediate scores.
\end{abstract}

\begin{abstract}
Aims To establish whether facial expression recognition is impaired in unaffected siblings of patients.
\end{abstract}

\section{Method Emotion and gender recognition were evaluated in a three- group pre-post study design in drug- naive patients with first-episode schizophrenia $(n=40)$ and their unaffected siblings ( $n=30$ ) compared with controls $(n=26)$.}

\section{Results Patients and their healthy siblings showed impaired emotion recognition but normal gender recognition compared with controls. Patients' performance did not improve despite effective clinical stabilisation.}

Conclusions Impaired performance in healthy siblings and time stability in patients provides evidence of impairment of facial emotion recognition as an actual phenotype of schizophrenia.

Declaration of interest None.
A key component of social cognition, facial expression recognition, is one of the hallmark deficits of schizophrenia (Schneider et al, 2006). Although people with schizophrenia may show deficits in other aspects of face perception (Hall et al, 2004), only expression recognition performance has been related to social dysfunction (Hooker \& Park, 2002). Studies have implicated interaction between the amygdala and prefrontal and temporal cortex in facial expression processing (Krolak-Salmon et al, 2004). In schizophrenia this deficit has been related to amygdala-hippocampal complex (AHC) dysfunction (Gur et al, 2002), although the frontal and temporal cortex may also have a role (Streit et al, 2001). Many of these regions, including the amygdala and the prefrontal and superior temporal cortices, are known to be reduced in volume in schizophrenia - see reviews by Lawrie \& Abukmeil (1998) and Wong \& Van Tol (2003) and the meta-analysis by Wright et al (2000). Interestingly, despite a recent contradictory result (McDonald et al, 2006), reduced volume of the AHC has been consistently reported in healthy siblings of patients (Lawrie et al, 1999; Aleman \& Kahn, 2005). However, some methodological issues (inclusion criteria, regions of interest, medial temporal lobe considered as a whole) could account for these discrepancies. Moreover, siblings show poor perception of non-verbal social-emotional cues (Toomey et al, 1999). Facial expression recognition is one of the most crucial vectors of non-verbal communication relying on amygdala integrity and may therefore be impaired in healthy relatives of patients, although this has never been evidenced.

Our hypothesis was that facial expression recognition performance, like AHC volume, would be distributed along a continuum between controls and patients with schizophrenia, with healthy relatives of patients having intermediate values, as has been shown for several cognitive (Saoud $e t$ al, 2000; Brunelin et al, 2007) and social functions (Toomey et al, 1999). Moreover, as previously reported with other cognitive deficits, patients are unlikely to perform as well as controls even when the disorder is effectively treated (Brunelin et al, 2007). To test this hypothesis we measured facial emotion recognition in patients experiencing their first schizophrenic episode before and after first antipsychotic treatment and in their healthy siblings compared with controls. The method used in the current study is original in using morphing controlling for performance in another task requiring facial feature analysis, that is gender recognition (Bediou et al, 2005). Our previous report of patients' preserved performance in this task suggests that it does not rely on the same dysfunctional structures.

\section{METHOD}

Forty drug-naive patients (mean age 26.9 years, s.d.=5.4) experiencing their first episode of schizophrenia diagnosed according to DSM-IV criteria (American Psychiatric Association, 1994), 30 of their non-affected first-degree relatives (mean age 30.4 years, s.d. $=10.4$ ) and 26 healthy controls (mean age 24.3 years, s.d.=3.3) were enrolled in this study. All participants were male. Their vision was normal or corrected to normal. Groups differed significantly in age $\left(F_{(2,91)}=5.12, P<0.01\right)$ and in years of fulltime education $\left(F_{(2,91)}=26.03, P<0.001\right)$. All participants received detailed information regarding the study and gave their written informed consent. The study was conducted in accordance with the latest version of the Declaration of Helsinki and its design was approved by the standing ethics committee. Recruitment took place between September 2003 and September 2005. Patients with schizophrenia were recruited at the Ibn Nafis psychiatric hospital in Marrakech. All participants were Moroccans, born, raised and currently living in Morocco. The group of healthy relatives consisted of unaffected siblings (brothers) of patients participating in the study; they had no past or current history of any Axis I DSM-IV disorder as verified by the Mini International Neuropsychiatric Interview (MINI; Sheehan et al, 1998) semi-standardised evaluation, version 4.4. Participants in the healthy control group also had no personal or familial past or current history of Axis I DSM-IV disorder 
as tested by MINI evaluation; they were recruited from the hospital staff and among students from the Medical and Pharmaceutical University of Marrakech. None had ever received psychotropic medication and none reported any misuse of drugs.

To address time stability and treatment effect of facial emotion recognition deficit in schizophrenia, participants in the schizophrenia group were tested twice, at baseline and after clinical stabilisation of symptoms under haloperidol treatment (mean dose $10.0 \mathrm{mg}$, s.d.=1.6). To control for potential learning or training effects, the other two study groups were also tested twice over a similar period. The mean interval between baseline and follow-up testing was 30.2 days $($ s.d. $=5.3$ ) in each group. Testing sessions consisted of each participant performing the expression and gender recognition task described below, the order being counterbalanced across participants and, for each participant, between testing sessions. At the time of each testing, symptoms were assessed by a trained clinician masked to participants' status. Patients' schizophrenic symptoms were assessed with the Positive and Negative Syndrome Scale (PANSS; Kay et al, 1987). Since depression may affect emotion recognition performance, depressive symptoms were also controlled for in the sibling and schizophrenia groups with the Calgary Depression Scale for Schizophrenia (CDSS; Addington et al, 1990) and in the control group with the abbreviated version of the Beck Depression Inventory (BDI; Beck et al, 1961). Schizotypal personality traits were evaluated in the sibling and control groups with the Schizotypal Personality Questionnaire (SPQ; Raine, 1991). Demographic and clinical data are summarised in Table 1.

\section{Tasks}

We used sensitive measures of emotional facial expression recognition and a control measure of facial gender recognition, both using morphed faces. A detailed description of the method can be found in our previous work (Bediou et al, 2005).

\section{Emotional facial expression recognition}

Briefly, 132 faces from two male and two female faces morphed between a neutral and an emotional expression (happiness, disgust, fear, anger) in $10 \%$ steps were each randomly presented on a computer screen for $1000 \mathrm{~ms}$, preceded by a fixation cross $(250 \mathrm{~ms})$ and followed by the choice list (neutral, happiness, disgust, fear or anger), which was maintained on the screen until the participant responded. Participants were required to match each face with the word that best described the emotional expression displayed by pressing the corresponding key.

\section{Facial gender recognition}

In the gender recognition task 132 faces, morphed between an average face with 'no gender' (obtained by averaging 20 male and 20 female faces in equal proportion) and one of six male or one of six female faces in $10 \%$ steps, were each randomly presented for $1000 \mathrm{~ms}$, preceded by a fixation cross $(250 \mathrm{~ms})$ and followed by the choice list (male or female). Participants were instructed to match each face with the word that best described its gender by pressing the corresponding key.

\section{Statistical analyses}

Dependant variables were the percentage of correct responses in the emotion and gender recognition tasks. Independent variables were the group (schizophrenia, siblings or control); the phase (baseline or follow-up); the task (expression or gender); the intensity of emotional expression or gender $(0$ $100 \%$ in $10 \%$ increments); the expression (happiness, disgust, fear or anger); and the gender (male or female). The first variable is an intra-individual factor whereas the others are inter-individual factors.

Performance of the schizophrenia group, time stability/treatment effect and performance of the siblings group were tested by means of repeated-measures analyses of variance; a main 3 group $\times 2$ phase $\times 2$ task $\times 10$ intensities multivariate analysis of variance (MANOVA) was followed by a 3 group $\times 2$ phase $\times 4$ expression $\times 10$ intensities and a 3 group $\times 2$ phase $\times 2$ gender $\times 10$ intensities MANOVA. To correct for multiple comparisons, Bonferroni correction led to a level of significance retained at 0.01 for analyses of variance (ANOVAs). Significant effects and interactions were then tested by means of planned comparisons using single-factor ANOVAs for interindividual factors and contrast analyses

Table I Characteristics of the sample (values are means and standard deviation)

\begin{tabular}{|c|c|c|c|c|c|}
\hline & \multicolumn{2}{|c|}{$\begin{array}{l}\text { Schizophrenia group } \\
\qquad(n=40)\end{array}$} & \multirow[t]{2}{*}{$\begin{array}{l}\text { Siblings group } \\
\qquad(n=30)\end{array}$} & \multirow[t]{2}{*}{$\begin{array}{l}\text { Control group } \\
\qquad(n=26)\end{array}$} & \multirow[t]{2}{*}{$P^{\prime}$} \\
\hline & Baseline & Follow-up & & & \\
\hline Age, years & $27.3(5.8)$ & & $31.2(10.6)$ & $24.3(3.3)$ & $<0.01$ \\
\hline Educational level, years & $9.6(2.1)$ & & $9(6.6)$ & $16.2(I . I)$ & $<0.001$ \\
\hline \multicolumn{6}{|l|}{ PANSS score } \\
\hline Positive (max. 49) & $29.5(7.1)$ & $10.2(6.7)$ & & & $<0.001$ \\
\hline Negative (max. 49) & $27.2(7.6)$ & II.4 (3.8) & & & $<0.001$ \\
\hline General (max. II2) & $39(6.6)$ & $20(4)$ & & & $<0.001$ \\
\hline CDSS (max. 27) & $\mathrm{I} .4(2.2)$ & $0.1(0.7)$ & $0.6(2.0)$ & & $<0.001$ \\
\hline $\mathrm{SPQ}(\max .74)$ & & & I4.I (13.5) & $10.8(11.7)$ & NS \\
\hline BDI (max. 39) & & & & $1.9(3.2)$ & \\
\hline Antipsychotic treatment, $\mathrm{mg}$ haloperidol & & $9.22(1.5)$ & & & \\
\hline
\end{tabular}

BDI, Beck Depression Inventory; CDSS, Calgary Depression Scale for Schizophrenia; max., maximum; SPQ, Schizotypal Personality Questionnaire.

I. Between-group comparisons were performed with single-factor analyses of variance for age and education level and with Student's $t$-tests for SPQ scores. Within-group comparisons for PANSS and CDSS scores were assessed with paired $t$-tests. 
using the full data-set for intra-individual factors (e.g. phase effects: baseline $v$. follow-up). Student's $t$-tests were used to probe group differences in socio-demographic characteristics. Given the significant group differences in age and education, and since performance did not correlate with either of these variables, repeated-measures analyses of covariance (MANCOVA) were also performed to control for a potential influence of these variables on significant effects. Since the same significant effects and interactions were found with MANOVAs and corresponding MANCOVAs, only results from the MANOVAs are reported.

\section{RESULTS}

Positive, negative and general schizophrenia symptoms as well as depressive symptoms were significantly reduced between baseline and follow-up in the patient group (Table 1).

\section{Emotion and gender recognition performance}

The main MANOVA revealed a main effect of group $\left(F_{(2,186)}=28.97, P<0.001\right)$, task $\left(F_{(1,186)}\right.$ $=777.86, P<0.001)$ and intensity $\left(F_{(9,1674)}\right.$ $=917.27, P<0.001)$ and significant group $\times$ task $\left(F_{(2,186)}=31.75, P<0.001\right)$, group $\times$ intensity $\quad\left(F_{(18,1674)}=10.51, \quad P<0.001\right)$, task $\times$ intensity $\left(F_{(9,1674}=105.14, P<0.001\right)$ and group $\times$ task $\times$ intensity interactions $\left(F_{(18,1674)}=6.86, P<0.001\right)$. There was no main effect of phase nor any interaction of phase with group, task or intensity. Consistent with our hypothesis, performance did not differ significantly between baseline and follow-up in any of the groups. There was a difference between overall emotion and gender recognition performance in all groups, suggesting that the emotion task was more difficult: emotion and gender recognition in the control group were $61 \%$ (s.d. $=9$ ) and $78 \%$ (s.d.=7) respectively. However, this effect was modulated by group. The schizophrenia group performed significantly worse than the control group on emotion recognition at baseline $\left(F_{(1,186)}=38.76, P<0.001\right)$ and the difference remained significant at follow-up $\left(F_{(1,186)}=38.16, P<0.001\right)$ despite clinical stabilisation of symptoms. Moreover, the siblings group performed significantly worse than the control group but significantly better than the schizophrenia group in overall facial expression recognition
(Fig. 1; scores averaged across all emotions and phases). No difference was found between groups on overall gender recognition $\left(F_{(1,186)}=1.06\right.$, NS). This absence of group difference was confirmed at all intensities of the gender morphs.

Analysis by emotion category revealed significant group $\times$ expression $\left(F_{(6,558)}=\right.$ 3.69, $P<0.001)$ and group $\times$ expression $\times$ intensity interactions $\quad\left(F_{(54,5022)}=1.50\right.$, $P=0.01$ ). Performance differed between the schizophrenia and control groups in the recognition of all basic emotions and between the sibling and control groups in the recognition of disgust and fearful faces (Fig. 2). Correlations between facial expression recognition and age $(r=-0.029$, $P=0.88)$ and between facial expression recognition and educational level $(r=0.075$, $P=0.71$ ) were not significant. Likewise, emotion recognition performance did not correlate with mood (assessed with the BDI) in the control group, nor with depression scores (assessed with the CDSS) in the sibling and schizophrenia groups.

\section{DISCUSSION}

Consistent with our hypothesis, emotion recognition performance was distributed along a continuum between the control group and the schizophrenia group, with the siblings group midway between, whereas gender recognition was highly preserved in all groups. Such impairment may reflect a specific deficit in emotion processing rather than a general impairment in face processing. Thus, the continuum in emotional facial expression recognition parallels the one observed in cognition (Saoud et al, 2000; Sitskoorn et al, 2004;
Brunelin et al, 2007) and in perception of other non-verbal social-emotional cues (Toomey et al, 1999) as well as in AHC volume (Lawrie et al, 1999). However, there have been few published research studies in which participants with schizophrenia were not found to differ from healthy controls in their emotion recognition skills (Flack et al, 1997; Gur et al, 2002). These data and our results suggest that emotion recognition skills may occur on a continuum, and that individuals with schizophrenia, in general, tend to score lower on this continuum than their healthy siblings or healthy unrelated individuals.

Our results also revealed that facial expression recognition was already impaired at the onset of the illness, before treatment, and that performance remained impaired after 1 month of low-dose haloperidol treatment despite symptom stabilisation, suggesting trait-like features. Although age and educational level differed significantly between groups, it is unlikely that these factors account for the group differences observed here. Indeed, neither correlation nor comparison between MANOVA and MANCOVA results suggested an effect of age or education on emotion recognition. In addition, most previous studies failed to find any influence of these variables on performance.

Abnormal visual scanning of emotional faces may indirectly underlie some aspects of face recognition impairments in schizophrenia (Loughland et al, 2002). It has been related to amygdala function (Taylor et al, 2002; Adolphs et al, 2005). Evidence for the stability of these disturbances over time and illness progression points to their traitlike nature (Streit et al, 1997). Moreover,

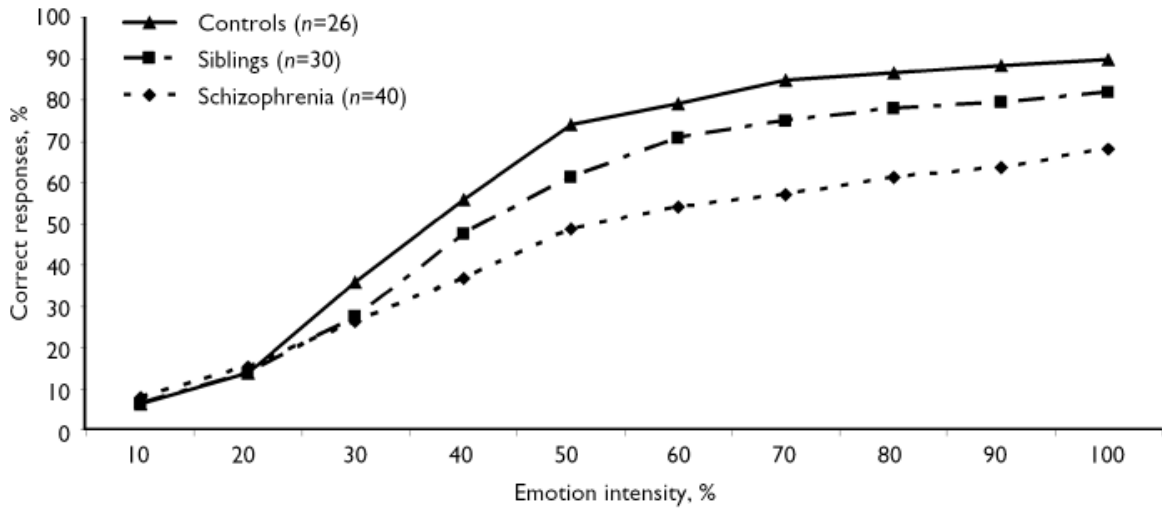

Fig. I Recognition of facial expressions as a function of emotion intensity. In the absence of phase effect or group $\times$ phase interaction, reported values are the mean between baseline and follow-up averaged among all emotions. Between-group differences according to single factor analysis of variance for the schizophrenia $v$. control and sibling v. control group comparisons are all significant $(P<0.00 \mathrm{I})$ at intensities of $30 \%$ and above. 


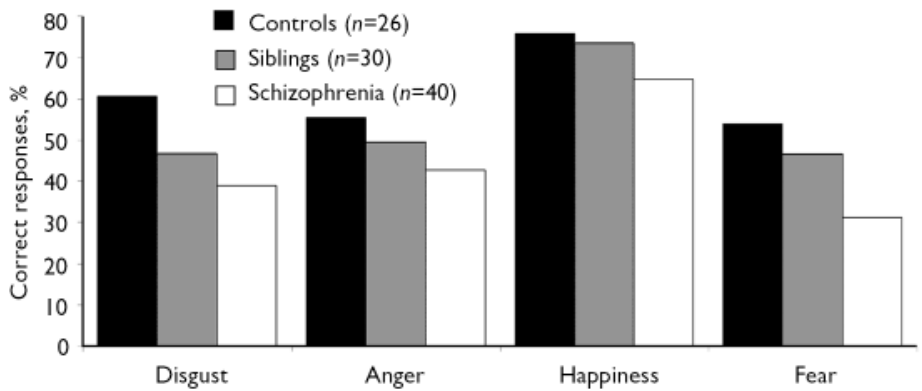

Fig. 2 Recognition of facial expressions of basic emotions by the control, sibling and schizophrenia groups. In the absence of phase effect or group $x$ phase interaction, reported values are the mean between baseline and follow-up averaged among all emotions. $P<0.001$, for all between-group differences according to single factor analysis of variance except for the difference between the sibling and control groups in recognition of fear $(P<0.0 \mathrm{I})$ and anger which was not significant $\left(F_{(1.186)}=3.48, P=0.063\right)$

abnormal visual scanning of facial expressions has also been documented in unaffected siblings of people with schizophrenia (Loughland et al, 2004), reinforcing the assumption that emotion recognition deficit can be considered as a potential marker of familial vulnerability to schizophrenia. Unlike expression recognition, gender recognition may rely on a more holistic face analysis that is achieved in posterior occipito-temporal areas despite abnormal visual scanning of face parts.

In contrast to the overall deficit in facial expression recognition in the schizophrenia group, unaffected siblings were only impaired in fear and disgust recognition, suggesting that the emotion-specific deficit of healthy relatives may generalise with illness onset. However, a comparable deficit in fear and disgust recognition had already been observed in participants whose schizophrenia was in remission in our previous study (Bediou et al, 2005). Atypical antipsychotic treatment, used in the latter study, may therefore be more efficient than haloperidol, used in the current study, in improving emotion recognition in schizophrenia (Kee et al, 1998). However, performance was not completely restored by either of these treatments and the deficit remains significant at all phases of the illness, supporting the trait-like hypothesis. Interestingly, relatives' performance appeared similar to that of patients when emotional expression was more subtle $(30 \%)$, whereas it was more like that of the control group at higher intensities of emotional expression $(80-90 \%)$. This may relate to the efficiency of social cognition processes (Toomey et al, 1999), facial scanning (Loughland et al, 2002), or both. However, this original result must be interpreted with caution. Indeed, even if the sibling and control groups did not differ significantly in anger recognition, lack of power might account for this result.

In social situations inaccurate decoding of emotional expression could be a source of stress and a barrier to deep social interactions and communication. It has been shown that metabolic and probably physical and social stress could cause an acute exacerbation of schizophrenia symptoms (van Os \& McGuffin, 2003). Thus, one can easily conceive that abnormal interpretation of emotional expressions could increase the level of emotional stress and thus participate in precipitating vulnerable people into schizophrenia. However, the neurochemistry of emotion recognition remains to be specified. There is growing evidence that dopamine has a central role in emotion recognition (Salgado-Pineda et al, 2005). Direct evidence comes from studies using pro-dopamine (Delaveau et $a l, 2005$ ) or anti-dopamine (Lawrence $e t$ $a l, 2002)$ agents in healthy volunteers. Also, indirect evidence is provided by studies of neuropsychiatric patients, specifically in schizophrenia (Bediou et al, 2005) and Parkinson's disease (Lachenal-Chevallet $e t$ al, 2006). Increasing serotonin transmission, on the other hand, can improve emotion recognition performance in healthy individuals (Harmer et al, 2003) and in people with major depressive disorder (Harmer et al, 2004). Abnormalities in the genes involved in dopamine transmission have been associated with poor cognitive functioning, reduced frontal lobe activation and high risk of schizophrenia (Goldberg et $a l, 2003)$, and variation in the serotonin transporter gene has been associated with variation in amygdala response to facial expressions (Hariri et al, 2002). This first report of emotion recognition as a marker of familial vulnerability to schizophrenia may encourage studies addressing its association with genetic polymorphism affecting dopamine or serotonin neurotransmission as well as the correlation between emotion recognition performance and amygdala volume. Moreover, given the strong relationship between emotion processing ability and functional outcome, specific remediation programmes should be encouraged (Wolwer et al, 2005). Likewise, the efficacy of emotion processing remediation programmes in vulnerable individuals as a putative preventive strategy should be evaluated.

\section{REFERENCES}

Addington, D., Addington, J. \& Schissel, B. (1990) A depression rating scale for schizophrenics. Schizophrenia Research, 3, 247-25I.

Adolphs, R., Gosselin, F., Buchanan, T.W., et al (2005) A mechanism for impaired fear recognition after amygdala damage. Nature, 433, 68-72.

Aleman, A. \& Kahn, R. S. (2005) Strange feelings: do amygdala abnormalities dysregulate the emotional brain in schizophrenia? Progress in Neurobiology, 77, 283-298.

American Psychiatric Association (1994) Diagnostic and Statistical Manual of Mental Disorders (4th edn) (DSM-IV). APA

Beck, A. T., Ward, C. H., Mendelson, M., et al (1961) An inventory for measuring depression. Archives of General Psychiatry, 4, 56I-57I.

Bediou, B., Krolak-Salmon, P., Saoud, M., et al (2005) Facial expression and sex recognition in schizophrenia and depression. Canadian Journal of Psychiatry, 50, 525-533.

Brunelin, J., d'Amato, T., Brun, P., et al (2007) Impaired verbal source monitoring in schizophrenia: an intermediate trait vulnerability marker? Schizophrenia Research, 89, 287-292.

Delaveau, P., Salgado-Pineda, P., Wicker, B., et al (2005) Effect of levodopa on healthy volunteers' facial emotion perception: an FMRI study. Clinical Neuropharmacology, 28, 255-261.

Flack, W. F., Cavallaro, L. A., Laird, J. D., et al (1997) Accurate encoding and decoding of emotional facial expressions in schizophrenia. Psychiatry, 60 197-210

Goldberg, T. E., Egan, M. F., Gscheidle, T., et al (2003) Executive subprocesses in working memory: relationship to catechol-O-methyltransferase Vall58Met genotype and schizophrenia. Archives of General Psychiatry, 60, 889-896.

Gur, R. E., McGrath, C., Chan, R. M., et al (2002) An fMRI study of facial emotion processing in patients with schizophrenia. American Journal of Psychiatry, 159, 19921999.

Hall, J., Harris, J. M., Sprengelmeyer, R., et al (2004) Social cognition and face processing in schizophrenia. British Journal of Psychiatry, 185, 169-170.

Hariri, A. R., Mattay, V. S., Tessitore, A., et al (2002) Serotonin transporter genetic variation and the response of the human amygdala. Science, 297, 400-403. 
Harmer, C. J., Bhagwagar, Z., Perrett, D. I., et al (2003) Acute SSRI administration affects the processing of social cues in healthy volunteers. Neuropsychopharmacology, 28, 148-152.

Harmer, C. J., Shelley, N. C., Cowen, P. J., et al (2004) Increased positive versus negative affective perception and memory in healthy volunteers following selective serotonin and norepinephrine reuptake inhibition. American Journal of Psychiatry, 161, 1256-1263.

Hooker, C. \& Park, S. (2002) Emotion processing and its relationship to social functioning in schizophrenia patients. Psychiatry Research, II2, 4|-50.

Kay, S., Fiszbein, A. \& Opler, L. (1987) The Positive And Negative Syndrome Scale (PANSS) for schizophrenia. Schizophrenia Bulletin, 13, 261-276.

Kee, K. S., Kern, R. S., Marshall, B. D., et al (1998) Risperidone versus haloperidol for perception of emotion in treatment-resistant schizophrenia: preliminary findings. Schizophrenia Research, 3I, 159-165.

Krolak-Salmon, P., Henaff, M. A., Vighetto, A., et al (2004) Early amygdala reaction to fear spreading in occipital, temporal, and frontal cortex: a depth electrode ERP study in human. Neuron, 42, 665-676.

Lachenal-Chevallet, K., Bediou, B., Bouvard, M., et al (2006) Emotional facial expression recognition impairment in Parkinson disease (in French). Psychologie et Neuropsychiatrie du Vieillissement, 4, 6I-67.

Lawrence, A. D., Calder, A. J., McGowan, S.W., et a (2002) Selective disruption of the recognition of facial expressions of anger. NeuroReport, I3, 88I-884.

Lawrie, S. M. \& Abukmeil, S. S. (1998) Brain abnormality in schizophrenia. A systematic and quantitative review of volumetric magnetic resonance imaging studies. British Journal of Psychiatry, 172, II0-120.

Lawrie, S. M., Whalley, H., Kestelman, J. N., et al (1999) Magnetic resonance imaging of brain in people at high risk of developing schizophrenia. Lancet, 353, 30-33.

Loughland, C. M., Williams, L. M. \& Gordon, E. (2002) Visual scanpaths to positive and negative facial emotions in an outpatient schizophrenia sample. Schizophrenia Research, 55, I59-170.

Loughland, C. M., Williams, L. M. \& Harris, A.W. (2004) Visual scanpath dysfunction in first-degree

BENOIT BEDIOU, PhD, University of Lyon, Centre Hospitalier Le Vinatier, Bron and Institut Fédératif des Neurosciences de Lyon, Bron, France; FATIMA ASRI, MD, University of Marrakech, Morocco: JEROME BRUNELIN, PhD, University of Lyon, Centre Hospitalier Le Vinatier and Institut Fédératif des Neurosciences de Lyon, Bron, France; PIERRE KROLAK-SALMON, MD, PhD, Institut National de la Santé et de la Recherche (INSERM) and Centre Hospitalier Le Vinatier, Bron; THIERRY D'AMATO, MD, PhD MOHAMED SAOUD, MD, PhD, University of Lyon, Centre Hospitalier Le Vinatier and Institut Fédératif des Neurosciences de Lyon, Bron, France; IMANE TAZI, MD, University of Marrakech, Morocco

Correspondence: Dr Benoit Bediou, CH Le Vinatier, University of Lyon, EA 3092, 95 Boulevard Pinel, 69677 Bron Cedex, France. Email: benoit.bediou@ch-le-vinatier.fr

(First received I7 July 2006, final revision I February 2007, accepted I5 February 2007)

relatives of schizophrenia probands: evidence for a vulnerability marker? Schizophrenia Research, 67, II-2I

McDonald, C., Marshall, N., Sham, P. C., et al (2006) Regional brain morphometry in patients with schizophrenia or bipolar disorder and their unaffected relatives. American Journal of Psychiatry, 163, 478-487.

Raine, A. (1991) The SPQ: a scale for the assessment of schizotypal personality based on DSM-III-R criteria. Schizophrenia Bulletin, 17, 555-564.

Salgado-Pineda, P., Delaveau, P., Blin, O., et al (2005) Dopaminergic contribution to the regulation of emotional perception. Clinical Neuropharmacolology, 28 228-237.

Saoud, M., d'Amato, T., Gutknecht, C., et al (2000) Neuropsychological deficit in siblings discordant for schizophrenia. Schizophrenia Bulletin, 26, 893-902.

Schneider, F., Gur, R. C., Koch, K., et al (2006) Impairment in the specificity of emotion processing in schizophrenia. American Journal of Psychiatry, 163, 442-447.

Sheehan, D.V., Lecrubier, Y., Sheehan, H., et al (1998) The Mini International Neuropsychiatric Interview (M.I.N.I.): the development and validation of a structured diagnostic interview for DSM-IV and ICD-10. Journal of Clinical Psychiatry, 59 (suppl. 20), 22-33.

Sitskoorn, M. M., Aleman, A., Ebisch, S. J., et al (2004) Cognitive deficits in relatives of patients with schizophrenia: a meta-analysis. Schizophrenia Research, 7I, 285-295.
Streit, M., Wolwer, W. \& Gaebel,W. (1997) Facial-affect recognition and visual scanning behaviour in the course of schizophrenia. Schizophrenia Research, 24, 31I-317.

Streit, M., loannides, A., Sinnemann, T., et al (2001) Disturbed facial affect recognition in patients with schizophrenia associated with hypoactivity in distributed brain regions: a magnetoencephalographic study. American Journal of Psychiatry, 158, 1429-1436.

Taylor, S. F., Liberzon, I., Decker, L. R., et al (2002) A functional anatomic study of emotion in schizophrenia Schizophrenia Research, 58, 159-172.

Toomey, R., Seidman, L. J., Lyons, M. J., et al (1999) Poor perception of nonverbal social-emotional cues in relatives of schizophrenic patients. Schizophrenia Research, 40, $12 \mid-130$

Van Os, J. \& McGuffin, P. (2003) Can the socia environment cause schizophrenia? British Journal of Psychiatry, 182, 291-292.

Wolwer, W., Frommann, N., Halfmann, S., et a (2005) Remediation of impairments in facial affect recognition in schizophrenia: efficacy and specificity of a new training program. Schizophrenia Research, 80 , 295-303.

Wong, A. H. \& VanTol, H. H. (2003) Schizophrenia: from phenomenology to neurobiology. Neuroscience and Biobehavioral Reviews, 27, 269-306.

Wright, I. C., Rabe-Hesketh, S., Woodruff, P. W., et al (2000) Meta-analysis of regional brain volumes in schizophrenia. American Journal of Psychiatry, 157, 16-25. 